\title{
Decision Model and Calculation of Human-job Matching Considering Risk Attitude in Uncertain Preference Order
}

\author{
Huimin Qiang ${ }^{1, *}$ Lipeng Wang $^{1}$ Min Huang $^{1}$ \\ ${ }^{1}$ School of Economics and Management, Jiangsu University of Science and Technology, Zhenjiang, Jiangsu, \\ China \\ *Corresponding author. Email: 769691107@qq.com
}

\begin{abstract}
The satisfaction of both operators and posts is an important basis for person-post matching. A decision-making method of person-post matching with uncertain preference order information was proposed considering the risk attitude of both parties. Firstly, the problem of person-post matching with uncertain preference information was described; then, according to the risk mapping function, the risk attitude factor of both parties was introduced to transform the uncertain preference information into an order value vector; then, the concept of person-post matching satisfaction was introduced and on this basis, considering the requirements of mutual satisfaction of the matching subjects of both parties, the optimal matching model of both parties' satisfaction was constructed. Finally, an example was given to verify the feasibility and effectiveness of the method.
\end{abstract}

Keywords: Management science, Person-post matching, Uncertain preference order, Risk attitude, Satisfaction.

\section{INTRODUCTION}

An earlier study of person-post matching was the H-R matching between interns and hospitals proposed by Roth A E [1]. Person-post matching is an important way for modern enterprises to realize the rational allocation of operating personnel and maximize economic benefits. Operators and posts are the two basic factors of enterprise production management. Reasonable person-post matching can effectively improve the job satisfaction, corporate performance, and competitiveness of the operators [2]. Therefore, how to achieve a reasonable match between posts is an important issue that enterprises urgently need to solve. Ertay $\mathrm{T}$ used data envelopment analysis to solve and determine the person-post matching problem of production line [3]; HUANG D K considered the interdependence between positions and the differences between selected employees, and a binary integer programming model with dual objectives was established to obtain the best personnel and position matching [4]. According to the matching problem based on complete order value information, Qi Yue proposed a matching decision method considering the psychological behavior of both subjects [5].

However, it needs to be pointed out that in the actual person-post matching problem, it is difficult to give accurate preference order information due to the influence of factors such as incomplete information grasp by both matching subjects, so the decision makers often give inaccurate and uncertain preference information of order interval [6]. However, there are few researches on person-post matching under uncertain preference order. Aiming at the interval number, language evaluation, $0-1$ judgment information and other forms of information under multiple evaluation indexes, $\mathrm{Xi}$ Chen established a person-post matching model with the goal of maximizing the total satisfaction of both subjects respectively [7]. In view of the matching problem under the uncertain preference order, Qi Yue constructed a muti-objective optimization model with the goal of maximizing the satisfaction of both matching subjects [8]. Based on the case that employee satisfaction is an uncertain preference order, Qian Yang established a decision model of person-post matching with the goal of 
maximizing and minimizing the difference between actual job satisfaction and expected job satisfaction of an employee [9].

The existing research provided some theoretical and methodological guidance for the person-post matching problem under uncertain preference order. However, the matching subjects of both sides were often considered to be completely rational, without considering the risk attitude in the process of matching in these studies. In the actual problem of job matching, most subjects are limited rational [10]. Therefore, aiming at the problem of person position matching under uncertain preference order, this paper proposed a decision-making method of person-post matching considering the risk attitude of both subjects.

\section{RELATED BASIC DEFINITIONS}

\subsection{Uncertain Preference Order}

Suppose $Z^{+}$is a set of positive integers, if $r=\left\{r^{L}, r^{L+1}, \ldots, r^{U}\right\}, r^{L}, r^{L+1}, \ldots, r^{U} \in Z^{+}$ and $r^{L} \leq r^{U}$, then $r$ is called the uncertain preference order [11]. ${ }^{r^{L}}, r^{U}$ are the lower limit and upper limit of $\tilde{\mathrm{r}}^{\text {respectively. When }} r^{L}=r^{U}$, the uncertain preference order $r$ degenerates to the order value. $r$ is a discrete set, denoted as $r=\left[r^{L}{ }_{,} r^{U}\right]$. For a subject's uncertain preference order, ${ }^{r}$ means that the subject ranks the other subject in positions $r^{L}$ to $r^{U}$.

\subsection{Risk Mapping Function}

For the uncertain preference order of a subject, the mapping

$$
\theta(r)=a(r)+\lambda b(r)
$$

is called the risk mapping function with the subject's risk attitude [12]. In (1), ${ }^{\lambda}$ is the risk attitude factor, representing the subject's risk attitude. Where $^{a(r)}$ is the midpoint value of the interval $\left[r^{L}, r^{U}\right]$, and $b(r)$ is the width of the interval. So ${ }^{\theta(r)}$ can be expressed as

$$
\theta(r)=0.5\left(r^{+}+r^{-}\right)+\lambda\left(r^{+}-r^{-}\right), \lambda \in[-0.5,0.5]
$$

\section{PROBLEM DESCRIPTION}

In the consideration of the person-post matching problem with uncertain preference order information, suppose the post set is $H=\left\{\square_{1}, \square_{2}, \ldots, \square_{\llbracket H]}\right\}$, where ${ }^{h_{i}}$ represents the matching subject ${ }^{i}$ in the post set, ${ }^{\|H\|}$ represents the number of posts, $i \in I=\{1,2, \ldots,|H|\}$ and the operator set is $E=\left\{e_{1}, e_{2}, \cdots, e_{\|E\|}\right\}$, where $e_{j}$ represents the matching operator $j$ in the post set, $|E|$ represents the number of operator; $j \in J=\{1,2, \ldots,|E|\} \quad$; let $\widetilde{W}_{i}=\left(\widetilde{w}_{i 1}, \widetilde{w}_{i 2}, \cdots, \widetilde{w}_{i|H|}\right)$ be the sequence vector of uncertain preferences given by job ${ }^{H_{i}}$ based on the information of the operator set ${ }^{E}$, and let $\tilde{P}_{j}=\left(\tilde{p}_{1 j}, \tilde{p}_{2 j}, \cdots, \tilde{p}_{|E| j}\right)$ be the uncertain preferences given by operator ${ }^{E_{j}}$ based on limited job information of post set $H$.

When the preference information about the operator ${ }^{E}$ given by the post $\square_{i}$ is uncertain preference order, consider the risk attitude of the decision maker in the matching process, according to definition B and (2), we can see that

$$
\theta\left(\tilde{w}_{i j}\right)=0.5\left(\tilde{w}_{i j}^{L}+\tilde{w}_{i j}^{U}\right)+\lambda_{H}\left(\tilde{w}_{i j}^{U}-\tilde{w}_{i j}^{L}\right)
$$

And the same

$$
\theta\left(\tilde{p}_{i j)}=0.5\left(\tilde{p}_{i j}^{L}+\tilde{p}_{i j}^{U}\right)+\lambda_{E}\left(\hat{p}_{i j}^{U}-\hat{p}_{i j}^{L}\right)\right.
$$

Let ${ }^{\square_{i}}$ sort ${ }^{e_{j}}$ according to the size of $\theta\left(\widetilde{w}_{i j}\right)$, then the uncertain sequence value vector $\widetilde{W}_{i}$ can be transformed into the sequence value vector $W_{i}=\left(w_{i 1}, w_{i 2}, \cdots, w_{i|H|}\right)$. In the same way, the uncertain sequence value vector $\widetilde{P}_{j}$ can be transformed into the sequence value vector $P_{j}=\left(p_{1 j}, p_{2 j}, \cdots, p_{|E| j}\right)$

\section{DECISION METHOD}

\subsection{Matching Satisfaction}

Assuming that ${ }^{\alpha_{i j}}$ is the satisfaction degree of post ${ }^{h_{i}}$ to ${ }^{e_{j}}$, and $\beta_{i j}$ is the satisfaction degree of 
operator $e_{j}$ to $\square_{i i}$, the satisfaction degree ${ }^{\alpha_{i j}}$ and $\beta_{i j}$ can be expressed as

$$
\begin{aligned}
& \alpha_{i j}=f\left(w_{i j}\right), i \in I_{j} j \in J_{(5)} \\
& \beta_{i j}=g\left(p_{i j}\right), i \in I_{s} j \in J
\end{aligned}
$$

It can be seen from generality that if operator $e_{j}$ ranks post $\square_{i}$ first, that is $\tilde{p}_{i j}=[1,1]$, it means that $e_{j}$ has the highest satisfaction with $\square_{\bar{i}}$; if operator $e_{j}$ ranks post $\square_{\bar{i}}$ last, that is $\tilde{p}_{i j}=[|H|,|H|]$, It shows that ${ }^{e_{j}}$ has the lowest satisfaction with $\square_{i}$. The satisfaction function constructed in this paper is as follows:

$f(x)=\left(\frac{|E|-x}{|E|-1}\right)^{2}$
$g(y)=\left(\frac{|H|-y}{|H|-1}\right)^{2}$

\subsection{Modeling}

According to (5) and (6), the order vector $W_{i}$ and $P_{j}$ can be transformed into satisfaction matrix $\left[\alpha_{i j}\right]_{(|H| \times|E|)}$ and $\left[\beta_{i j}\right]_{(|H| \times|E|)}$. Let $x_{i j}$ be a variable from 0 to 1 . When $x_{i j}=0$, it means that the operator $e_{j}$ does not match the post $\square_{i}$; when $x_{i j}=1$, it means that the operator ${ }^{e_{j}}$ matches the post $\square_{i}$. Therefore, the person-post matching decision model is established as follows:

$$
\begin{aligned}
& \max \quad Z_{1}=\sum_{i=1}^{\mid H \|} \alpha_{i j} x_{i j}(9) \\
& \max \quad Z_{2}=\sum_{j=1}^{\mid E \|} \beta_{i j} x_{i j}(10) \\
& \text { s.t. } \quad \sum_{j=1}^{\|E\|} x_{i j}=1, i=1,2, \cdots,|H| \\
& \sum_{i=1}^{\|H\|} x_{i j} \leq 1, j=1,2, \ldots,\|E\| \\
& x_{i j}=0 \quad \text { or } 1
\end{aligned}
$$

In this model, (9) and (10) are the objective functions of the model. (9) and (10) respectively mean to maximize the total degree of operator's satisfaction with job matching and maximize the total degree of operator's satisfaction with job matching. (11) indicates that each post must and can only be matched with one operator; (12) indicates that each operator is matched with at most one post.

\subsection{Model Solution}

Since the objective function has the same dimensional order, linear weighting method is used to weight the (9) and (10). Assuming that ${ }^{\omega_{1}}$ and $\omega_{2}$ respectively represent the weights of objectives $Z_{1}$ and $Z_{2}, 0<\omega_{1}<1,0<\omega_{2}<1$, and satisfy $\omega_{1}+\omega_{2}=1$, then the dual objective optimization model can be transformed into a single objective optimization model:

$\max \quad Z=\sum_{i=1}^{|H|} \sum_{j=1}^{\llbracket E \|} \gamma_{i j} x_{i j}(14)$

s.t. $\quad \sum_{j=1}^{\mid E \|} x_{i j}=1, i=1,2, \ldots,|H|$

$\sum_{i=1}^{\|H\|} x_{i j} \leq 1, j=1,2, \ldots, \| E \mid$

$x_{i j}=0$ or $1_{(17)}$

In the target (14), $\gamma_{i j}=\omega_{1} \alpha_{i j}+\omega_{2} \beta_{i j}$, $i=1,2, \cdots,\|H\|, j=1,2, \cdots,|E|$, where $\omega_{1}$ and

$\omega_{2}$ reflect the importance of goal in actual personpost matching, they are generally given by both parties after negotiation, but in consideration of the fairness of both parties, let $\omega_{1}=\omega_{2}$. When there are many variables and constraint conditions in the model, software such as Lingo11.0 and MATLAB can be used, or heuristic algorithm and genetic algorithm can be used to solve. According to the solution results of the model, the optimal humanpost matching scheme considering the risk attitude of the subject can be obtained under the condition of uncertain preference order.

\section{EXAMPLE ANALYSIS}

An enterprise planned to recruit operators in line with the requirements of five posts $\left(\mathrm{H}_{1}, \mathrm{H}_{2}, \mathrm{H}_{3}, \mathrm{H}_{4}, \mathrm{H}_{5}\right)$, and finally eight operators $\left(E_{1}, E_{2}, E_{3}, E_{4}, E_{5}, E_{6}, E_{7}, E_{8}\right)$ had entered the final assessment. The decision-maker of the Department where the post was located evaluated and analyzed the eight operators from their operation skills, personality, creativity and teamwork ability, and gave the uncertain preference order of the overall evaluation information of different operators as shown in "Table 1"; similarly, different operators evaluated and analyzed five posts from the perspective of enterprise prospect, salary and welfare, enterprise future planning and enterprise working environment, and gave the uncertainty preference order of overall evaluation 
information of different posts, as shown in "Table $2 "$.

Table 1. The favorable value of operators given by decision-makers in different posts $\widetilde{w}_{i j}$

\begin{tabular}{|l|l|l|l|l|l|l|l|l|}
\hline \multirow{2}{*}{ Posts } & \multicolumn{7}{|l|}{ Operators } \\
\cline { 2 - 9 } & $\boldsymbol{E}_{1}$ & $\boldsymbol{E}_{2}$ & $\boldsymbol{E}_{\mathbf{3}}$ & $\boldsymbol{E}_{4}$ & $\boldsymbol{E}_{5}$ & $\boldsymbol{E}_{5}$ & $\boldsymbol{E}_{7}$ & \multicolumn{1}{|l|}{$\boldsymbol{E}_{8}$} \\
\hline $\boldsymbol{H}_{\mathbf{1}}$ & {$[7,8]$} & {$[4,4]$} & {$[1,2]$} & {$[3,5]$} & {$[1,3]$} & {$[2,3]$} & {$[4,6]$} & {$[5,8]$} \\
\hline $\boldsymbol{H}_{\mathbf{2}}$ & {$[4,6]$} & {$[1,2]$} & {$[5,7]$} & {$[2,3]$} & {$[6,8]$} & {$[2,4]$} & {$[5,7]$} & {$[3,4]$} \\
\hline $\boldsymbol{H}_{\mathbf{I}}$ & {$[1,3]$} & {$[3,5]$} & {$[2,3]$} & {$[7,7]$} & {$[6,7]$} & {$[4,7]$} & {$[2,5]$} & {$[1,3]$} \\
\hline $\boldsymbol{H}_{4}$ & {$[2,3]$} & {$[6,8]$} & {$[4,5]$} & {$[1,4]$} & {$[5,8]$} & {$[5,6]$} & {$[6,7]$} & {$[2,5]$} \\
\hline $\boldsymbol{H}_{\mathbf{5}}$ & {$[5,6]$} & {$[2,3]$} & {$[8,8]$} & {$[5,8]$} & {$[1,3]$} & {$[4,7]$} & {$[3,6]$} & {$[5,5]$} \\
\hline
\end{tabular}

Table 2 . The favorable value of different positions given by different operators $\tilde{p}_{i j}$

\begin{tabular}{|c|c|c|c|c|c|c|c|c|}
\hline \multirow{2}{*}{ Posts } & \multicolumn{8}{|c|}{ Operators } \\
\hline & $E_{1}$ & $E_{2}$ & $E_{3}$ & $E_{4}$ & $E_{5}$ & $E_{6}$ & $\boldsymbol{E}_{\bar{\gamma}}$ & $E_{8}$ \\
\hline$H_{1}$ & {$[1,3]$} & {$[4,5]$} & {$[2,4]$} & {$[1,2]$} & {$[3,4]$} & {$[1,4]$} & {$[5,5]$} & {$[3,5$} \\
\hline$H_{2}$ & {$[4,5]$} & {$[2,5]$} & {$[2,3]$} & {$[1,4]$} & {$[1,3]$} & {$[3,4]$} & {$[3,4]$} & {$[2,3$} \\
\hline$H_{\Omega}$ & {$[2,3]$} & {$[1,1]$} & {$[3,5]$} & [2.4] & {$[5,5]$} & {$[3,4]$} & {$[1,2]$} & {$[4,5$} \\
\hline $\mathrm{H}_{4}$ & {$[3,5]$} & {$[2,3]$} & {$[2,4]$} & {$[3,4]$} & {$[4,4]$} & {$[1,2]$} & {$[2,5]$} & {$[1,2$} \\
\hline$H_{5}$ & {$[1,2]$} & {$[3,4]$} & {$[4,5]$} & {$[2,5]$} & {$[2,4]$} & {$[2,3]$} & {$[4,5]$} & {$[3,4]$} \\
\hline
\end{tabular}

After investigation, it was found that the post $H$ was risk preference, while the operator ${ }^{E}$ was risk aversion, so let $\lambda_{H}=0.2, \lambda_{E}=-0.2$. Then, according to the satisfaction functions $f(x)=\left(\frac{|8|-x}{7}\right)^{2}$ and $g(y)=\left(\frac{\sqrt{5 \mid-y}}{4}\right)^{2}$, the job satisfaction matrix $\left[\alpha_{i j}\right]_{(5 \times 8)}$ and the operator satisfaction matrix $\left[\beta_{i j}\right]_{(5 \times 8)}$ could be calculated.

Considering the fairness of the matching between the post and the operator, it may be assumed that $\omega_{1}=\omega_{2}=0.5, \quad$ and $\gamma_{i j}=0.5 \alpha_{i j}+0.5 \beta_{i j} \quad, \quad i=1,2,3,4,5$, $j=1,2,3,4,5,6,7,8$. Therefore, the coefficient matrix of the objective function could be calculated, as shown in "Table 3". Therefore, using Lingo 11.0 to solve the model could be obtained:

$$
x_{13}=x_{22}=x_{37}=x_{48}=x_{55}=1 \text {, the }
$$
remaining $x_{i j}=0$

Thus, the optimal solution for job matching was obtained: $\left(H_{1}, E_{3}\right),\left(H_{2}, E_{2}\right),\left(H_{3}, E_{7}\right),\left(H_{4}, E_{8}\right)$, $\left(H_{5}, E_{5}\right)$. In this example, if the risk attitudes of both parties were not considered, that was, $\lambda_{H}^{y}=\lambda_{E}^{z}=0$, the matching satisfaction matrix of 
both parties $\left[\alpha_{i j}^{\sigma}\right]_{5 \times 8}$ and $\left[\beta_{i j}^{\prime}\right]_{5 \times 8}$ was obtained.

Considering the fairness of the matching

Table 3. Coefficient matrix

\begin{tabular}{|l|l|l|l|l|l|l|l|l|}
\hline$Y_{i j}$ & $E_{1}$ & $E_{\mathbf{2}}$ & $E_{\mathbf{2}}$ & $E_{4}$ & $E_{\mathbf{5}}$ & $E_{6}$ & $E_{\bar{\gamma}}$ & $E_{8}$ \\
\hline$H_{\mathbf{1}}$ & 0.50 & 0.16 & 0.78 & 0.59 & 0.49 & 0.38 & 0.04 & 0.04 \\
\hline$H_{\mathbf{2}}$ & 0.26 & 0.63 & 0.54 & 0.49 & 0.51 & 0.29 & 0.17 & 0.45 \\
\hline$H_{\mathbf{I}}$ & 0.63 & 0.66 & 0.49 & 0.04 & 0.04 & 0.12 & 0.76 & 0.50 \\
\hline$H_{4}$ & 0.53 & 0.28 & 0.45 & 0.37 & 0.04 & 0.59 & 0.32 & 0.76 \\
\hline$H_{\mathbf{5}}$ & 0.37 & 0.40 & 0.03 & 0.29 & 0.78 & 0.32 & 0.20 & 0.38 \\
\hline
\end{tabular}

Table 4. Person-post matching results under different circumstances

\begin{tabular}{|l|l|}
\hline Person-post matching scheme & Results \\
\hline$\lambda_{H}=0.2, \lambda_{\mathrm{F}}=-0.2$ & $x_{13}=x_{22}=x_{17}=x_{48}=x_{55}$ \\
\hline$\lambda_{H}^{r}=\lambda_{\mathrm{E}}^{r}=0$ & $x_{13}=x_{24}=x_{27}=x_{48}=x_{56}$ \\
\hline
\end{tabular}

Between the post and the operator, it may be assumed that $\omega_{1}=\omega_{2}=0.5$, and $\gamma_{i j}=0.5 \omega_{1}^{*}+0.5 \omega_{2}^{*}$. Similarly, using Lingo 11.0 to solve the model could be obtained:

$\mathrm{x}_{13}=\mathrm{x}_{24}=\mathrm{x}_{37}=\mathrm{x}_{48}=\mathrm{x}_{56}=1$, the remaining $\mathrm{x}_{\mathrm{ij}}=0$

Thus, the optimal solution for job matching was obtained: $\left(H_{1}, E_{3}\right),\left(H_{2}, E_{4}\right),\left(H_{3}, E_{7}\right),\left(H_{4}, E_{8}\right)$, $\left(H_{5}, E_{6}\right)$

"Table 4" showed the results of person-post matching when the risk attitudes of both parties were considered or not considered. It could be seen that the matching results obtained under different circumstances were not the same exactly, which indicated that the risk attitudes of both parties had a certain influence on the person-post matching results.

\section{CONCLUSION}

Aiming at person-post matching problem with uncertain preference orderings, considered the risk of operator and post, uncertain preference information could be converted to vector sequence values, and was introduced into the matching degree of satisfaction function, based on this, a person-post matching decision method considering risk attitude under uncertain preference order information was proposed, and the following main conclusions were obtained.

This paper considered the risk attitude of both parties in person-post matching, and introduced it into the research of person-post matching decisionmaking, which provided a new idea for the followup research on person-post matching theory and methods;

People are generally bounded rationality in the person-post matching problem; the risk attitude of both parties will affect the result to a certain extent. The decision-making method proposed in this paper could better reflect the decision-making behavior in the person-post matching.

Therefore, this method considered the risk attitude of both parties in person-post matching, and provided a solution for solving the man-post matching problem, which had certain theoretical and practical application value.

\section{AUTHORS' CONTRIBUTIONS}

Huimin Qiang conceived of the study, designed the study, and analyzed the data. All authors contributed to the writing and revisions.

\section{REFERENCES}

[1] Roth A E. On the allocation of resident to rural hospitals: a general property of two-sided 
matching markets [J]. Econometrica, 1986, 54(2): 425-427.

[2] Dobrila P, Alejandra D, Sanja P. Decision 'support tool for multi-objective job shop scheduling problem with linguistically quantified decision functions [J]. Decision Support Systems, 2007,43(4) :1527-1538.

[3] Ertay T, Da R. Data envelopment analysis based on decision model for optimal operator allocation in CMS [J]. European Journal of Operational Research, 2005, 164(3):800-810.

[4] HUANG D K, CHIU H N, YEH R H, et al. A fuzzy multi-criteria decision-making approach for solving a bi-objective personnel assignment problem $[\mathrm{J}]$. Computers \& Industrial Engineering, 2009, 56(1):1-10.

[5] Qi Yue. Bilateral Matching Decision-Making Method Considering Subject's Psychological Behavior $[\mathrm{J}]$. Systems Engineering and Electronic Technology, 2013, 35(1): 120-125.

[6] Yanpin Jiang, Decai Kong, Duoning Yuan. Bilateral stable matching decision-making method with ordered interval preference information $[\mathrm{J}]$. System Engineering Theory and Practice, 2017,37(08):2152-2161.

[7] Xi Chen, Zhiping Fan. Research on Bilaterally Matching Job Seekers and Positions Considering Multiple Forms of Information [J]. Operations Research and Management, 2009,6(18): 103-109.

[8] Qi Yue, Zhiping Fan. Research on Bilateral Matching Decision-Making Problem with Uncertain Preference Order Information [J]. Operations Research and Management, 2012, 21(1): 57-63.

[9] Qian Yang, Hui Zheng, Zhichang Zhang. Man-post matching decision model and calculation based on uncertain preference order information [J]. Journal of $\mathrm{Xi}$ 'an University of Architecture and Technology (Natural Science Edition), 2014,46(04):609614.

[10] Kahneman D, Tversky A. Prospect theory: an analysis of decision under risk [J]. Econometrica, 1979, 47(2):263-291.

[11] Fan Z P, Yue Q, Feng B, et al. An approach to group decision-making with uncertain preference ordinals $[\mathrm{J}]$. Computers \& Industrial Engineering, 2010, 58(1):51-57.

[12] You T, Fan Z P. An Interval Number Multiindex Decision Making Method Based on Decision Maker's Risk Attitude [J]. Operations Research and Management, 2002, 11(5): 1-4. 